\title{
Perché così poca DP in Italia? Una risposta dalla Sicilia
}

\author{
da Flavia Caputo \\ Unità Operativa di Nefrologia e Dialisi, Sezione di Dialisi Peritoneale, \\ Ospedale Civico, Palermo
}

a Marco Lombardi

Unità Operativa di Nefrologia, Ospedale S. Maria Annunziata, Antella - Firenze

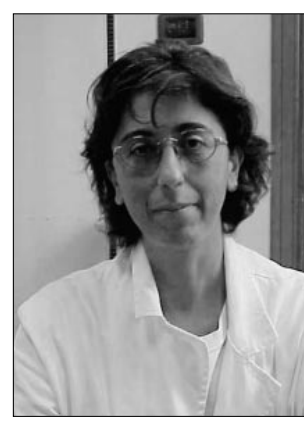

C aro Marco, se è vero, come tu dici, che in Italia nel 1998 solo il $10 \%$ dei pazienti ha utilizzato la dialisi peritoneale (DP) come tecnica sostitutiva della funzione renale e se questa prevalenza non è variata negli ultimi quindici anni, occorre ammettere che, almeno in Italia, la DP è effettivamente un trattamento di seconda scelta, per pazienti di seconda classe, curati da medici un po'speciali.

E se l'Italia rappresenta un tipico caso di difficile diffusione della DP, la Sicilia è tra le regioni italiane quella che con il suo $3.5 \%$ di pazienti in DP contribuisce maggiormente a mantenere questa condizione.

La nostra isola è, dunque, il paradigma di un ambiente poco favorevole alla penetrazione di questa pratica terapeutica e vorrei tentare, per quello che la nostra esperienza può insegnare, di dare una risposta alla tua domanda: "perché?"

Può capitare, per esempio, che un primario di nefrologia sia molto poco favorevole alla DP: se è consulente in qualche Centro di dialisi privato o se ne possiede uno, magari attraverso un congiunto, sarà naturalmente portato a privilegiare il trattamento extracorporeo (EC) rispetto a quello peritoneale; tenderà più $\mathrm{o}$ meno inconsciamente a non informare adeguatamente i potenziali candidati alla dialisi peritoneale, anzi li allarmerà e li impaurirà battendo sul tasto della "pericolosità" e "inefficienza" della peritoneale rispetto all'emodialisi; farà una selezione negativa, affidando alla DP i pazienti più compromessi, quelli che non hanno più alcuna possibilità di accesso vascolare o che

\section{Pazienti in Dialisi Peritoneale al Civico di Palermo 1981-2000 (aggiornati a Giugno)}

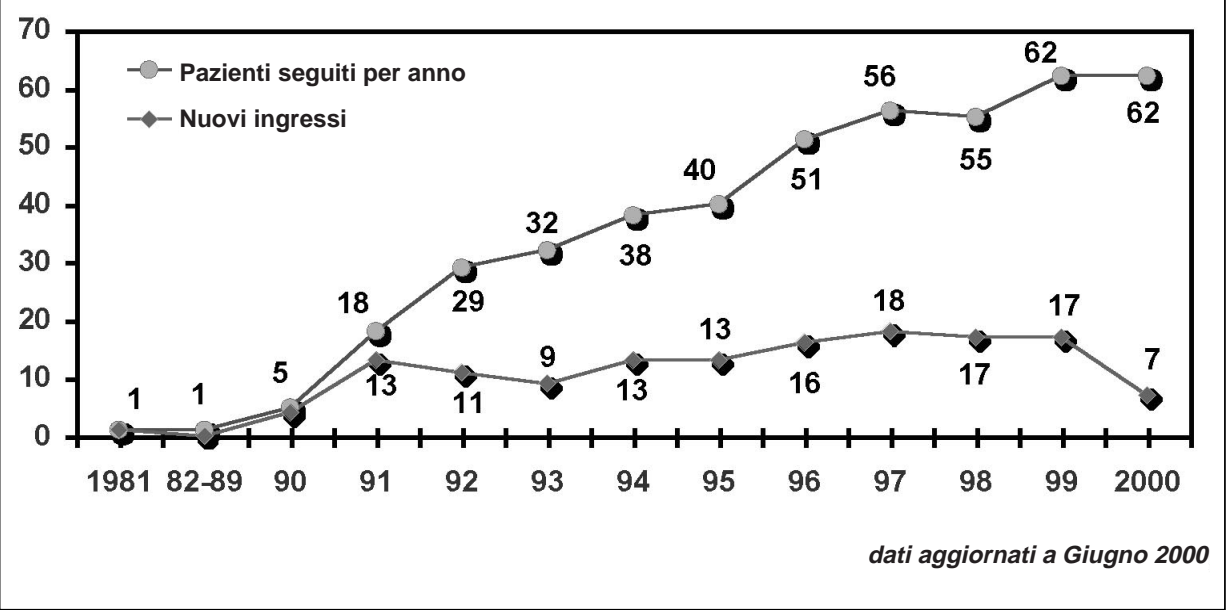


pillarmente sul territorio, e sono state (sono?) direttamente o indirettamente "collegate" a nefrologi che operano contemporaneamente nelle strutture ospedaliere, vuoi che possa attecchire e crescere la pianta della DP?

Non sempre, tuttavia, sono così palesi e grossolani interessi economici a impedire l'avvio di un programma di DP. Bisogna convincersi che proprio per il fatto che costa meno, la DP muove interessi meno corposi e meno diffusi; sono largamente inferiori a quelle della EC non solo le risorse destinate alla ricerca e alla diffusione delle conoscenze - che sono oggi più che mai largamente dipendenti dagli investimenti delle aziende produttrici ma anche le risorse dedicate alle innumerevoli e multiformi iniziative attraverso le quali l'industria manifatturiera cattura la "simpatia" degli operatori sanitari.

Il ruolo di freno che in Sicilia svolgono gli interessi del privato, possono benissimo altrove essere rappresentati da opportunità di trials, fondi di ricerca, visibilità dei risultati che in ultima analisi si traducono in consistenti vantaggi per chi vi accede.

Ma non c'è solo il vile metallo a deprimere le sorti della nostra "beneamata"; diciamocelo pure, una buona volta: la DP è meno friendly, richiede una presenza del medico accanto al paziente che non è nemmeno lontanamente paragonabile a quella dell'EC; ha un'incidenza di complicanze acute molto maggiore dell' $\mathrm{EC}$, è meno tecnologica, possiede poca elettronica, sembra proprio di serie B.

Questo spiega perché, fatte salve alcune "oasi felici" che costituiscono le eccezioni e non la regola, anche nelle altre regioni d'Italia (comprese quelle del pragmatico Nord-Est) esiste una specie di ostracismo alla DP, anche se di carattere più sfumato.

$\mathrm{Ci}$ vuole, dunque, quel piacere di stare accanto al malato e di armeggiare insieme con lui e di sfidare insieme con lui le leggi della biologia, per le quali il peritoneo non è una membrana di dialisi, per affezionarsi a questo mondo e per non dare spazio agli anticorpi anti DP che altrimenti crescono, crescono a vista d'occhio. Ecco perché molti si sono accostati anche con curiosità e interesse al mondo della DP e se ne sono ritratti, impauriti e delusi.

Infine i pazienti: non è vero che tutti amano gestire la propria malattia e non è vero che tutti chiedono di essere restituiti a una vita normale; ve ne sono - e sono anche molti - che si abbandonano, che chiedono di essere curati più che di curarsi, che desiderano essere guidati dentro la dialisi più che guidare la propria dialisi. La DP non si addice, evidentemente, a questo tipo di pazienti, né si addice, ahimé, alle migliaia di dializzati del Sud che difficilmente hanno un lavoro a prescindere dalla condizione di malattia e vivono di pensione di "invalidità".

\section{Che fare?}

Occorre prima di tutto e semplicemente accettare di essere minoranza e isola nel mare della extracorporea, lavorare con continuità e con pazienza per istruire noi stessi, i nostri infermieri, i nostri pazienti a gestire questo spazio di libertà che abbiamo contribuito a creare, per preservarlo dall'inutile vittimismo o dalla voglia di ergersi ad élite incompresa.

Occorre sapere ricercare i punti deboli della metodica e pazientemente provare e riprovare le soluzioni possibili per superarli.

Occorre, soprattutto, crederci fermamente: solo così anche in un luogo impervio e aspro come la Sicilia si farà lentamente crescere la pianta agile e tenace della DP.

Noi ci abbiamo creduto. Noi l'abbiamo fatto.

Siamo gran parte di quel 3.5\% di DP in Sicilia, siamo tra quelli che non intendono mettersi il cuore in pace.

flavia.caputo@tin.it 